\title{
Retail price regulation and innovation: Reference pricing in the pharmaceutical industry
}

\author{
D. Bardey ${ }^{\mathrm{a}, \mathrm{b}}$, A. Bommier ${ }^{\mathrm{c}}$, B. Jullien ${ }^{\mathrm{d}, *}$ \\ a University of Rosario (Bogota), Colombia \\ ${ }^{\mathrm{b}}$ Toulouse School of Economics (GREMAQ), France \\ c Toulouse School of Economics (CNRS, GREMAQ), France \\ d Toulouse School of Economics (IDEI and GREMAQ), Manufacture des Tabacs, 31000 Toulouse, France
}

\section{A R T I C L E I N F O}

\section{Article history:}

Received 25 June 2008

Received in revised form 1 November 2009

Accepted 11 November 2009

Available online 3 December 2009

\section{JEL classification:}

I18

L11

L15

L51

Keywords:

Innovation

Me-too

Reference pricing

Pharmaceutical laboratories

\begin{abstract}
A B S T R A C T
Our paper is a first attempt to evaluate the long run impact of reference pricing on pharmaceutical innovation, health and expenditures. The model is based on a dynamic game involving three types of agents: pharmaceutical firms, consumers and a regulatory entity. Pharmaceutical firms choose the level of research investment and its innovative content, then negotiate introductory prices for new drugs with the regulator. Reference pricing affects negatively the intensity of research and it also modifies the types of innovations that are brought to the market, deterring small innovations. The model is calibrated with a small data on statins in France. Our results suggest that reference pricing typically generates a decline in health, whereas discounted expenditures may decrease or increase, depending on the degree of deterrence of cost reducing innovations.
\end{abstract}

(c) 2009 Elsevier B.V. All rights reserved.

\section{Introduction}

A dramatic increase of pharmaceutical expenditures has been observed in most developed countries during the two last decades. In United States for example, spending on outpatient care grew by $17 \%$ in 1999 , whereas, total health expenditure only grew by 3.3\% during the same year (Danzon and Pauly, 2002). Over the last years, several countries - such as Germany, Italy or Australia - have tried to reduce health expenditure by adopting the principle of drug reimbursement based on therapeutic equivalence, a system referred to as therapeutic reference pricing. ${ }^{1}$ While modalities vary, the common feature of reference pricing regulations is that drugs are divided into different classes according to their active agents and/or their indications. Drugs reimbursement to the patients is then defined by class, based on a reference price, set according to

\footnotetext{
* Corresponding author. Tel.: +33 561128561.

E-mail address: bjullien@cict.fr (B. Jullien).

1 See Lopez-Casanovas and Puig-Junoy (2000), Danzon (2001) and Danzon and Ketchman (2004) for more details on reference pricing and its applications.
}

some domestic prices, usually with greater weight on low prices. ${ }^{2}$ In the short run, the product space is given and reference pricing mechanically generates a fall in drug reimbursement and prices. Instead, in a dynamic environment, where innovation by pharmaceutical firms is driven by the prospect of future profits, the impact of such a regulation is far from being obvious.

In a static framework, Brekke et al. (2007) emphasize the effect of different reference pricing modalities on the laboratories' profitability and consequently on new drugs' entry likelihood. By contrast, our paper is a first attempt to evaluate the long run equilibrium impact of reference pricing on pharmaceutical innovation, delays of introduction, health and expenditures. As expected, by reducing profitability, reference pricing regulations negatively affect the intensity of research and delay the introduction of new drugs. We point out that they also modify the types of innovations that are brought to the market due to a differential effect on the prices of drugs at various innovative levels. The

\footnotetext{
2 This is referred to internal reference pricing, as opposed to external reference pricing which relies on foreign prices.
} 
regulation may then divert resources from low margin/low innovation research projects toward higher margin projects. ${ }^{3}$ Based on the theoretical analysis and results of calibrated simulations, we emphasize mechanisms that were not understood before, showing that one has to be very cautious when predicting the impact of reference pricing on the long run, in particular for drug expenditures.

The analysis is conducted in a typical continental European context, where health insurance coverage is very high and where regulators have a strong degree of control over the retail prices of drugs. For this purpose we build a dynamic model of the pharmaceutical sector, inspired partly by the French system. ${ }^{4}$ The model involves three types of actors: producers (pharmaceutical firms), consumers (patients) and a regulator. Producers choose their R\&D investment in order to maximize their profit, anticipating future price and market sales dynamics. Moreover, laboratories can choose between two types of innovation: vertical innovation (or pioneer) and horizontal (also called me-too). Drug pricing, which plays a key role in the model, results from a bargaining game between the producers and the regulator who accounts for consumers' welfare. The resulting introductory price acts as a pricecap and depends on the innovative content of the drug and on the average prices of drugs already present on the market. Thus, although prices are individually negotiated with the regulator, they are subject to some form of competitive pressure: the presence of competing drugs limits the ability of an innovator to extract rents.

Even though the structure of the bargaining game is exogenous, the outcome may be affected by regulations that modify the reimbursement rules for patients and thus final market prices, such as reference pricing. We define a therapeutic class as the set of drugs that are horizontally differentiated and analyze the impact of reference pricing on the outcome of the bargaining process and the equilibrium. The direct effect of reference pricing regulation is that the prices of innovations within existing classes (me-too drugs) are pushed down compared to the case with no reference pricing. The introduction of me-too drugs is then delayed or even discouraged. The impact on the profitability of a pioneer drug is ambiguous: on one hand the duration of its monopoly position increases ${ }^{5}$, on the other hand the competition after entry of a me-too is intensified. The overall effect depends on the combination of the price and long run effects on innovation. To evaluate this effect we calibrate the model on the market for statins: we conclude that while the patients' health is always hurt, the discounted value of health expenditures may increase or decrease.

The paper is structured as follows. Section 2 presents the model, describing successively the retail market, the bargaining framework (with and without reference pricing) and the innovation sector. In order to address technical difficulties progressively the paper first focuses, in Sections 3 and 4, on the case where all innovations occur within the same class. More precisely, Section 3 discusses the price dynamics and Section 4 the innovation trajectories. Section 5 extends the analysis to the case where two verticaly differentiated therapeutic classes are considered. Section 6 discusses welfare consequences. Section 7 briefly presents the market for statins in France, that served to calibrate the model, and exposes the results from numerical simulations. Section 8 discusses some extensions of the model. Section 9 concludes.

\footnotetext{
3 The fact that reference pricing may alter the innovation process in favor of a "pioneer" drug, is mentioned in Lopez-Casanovas and Puig-Junoy (2000).

${ }^{4}$ As will be discussed later, our model can be translated to the US market context where health plans play a similar role to the regulator in our model.

5 Or its effective patent life (O'Donoghue et al., 1998).
}

\section{The base model}

The model involves three types of actors that intervene at different stages in a continuous time set-up: innovators/producers, the regulator and patients/doctors. At the upstream stage, innovators invest in R\&D and introduce new molecules or treatments. At the downstream stage, the regulatory agency and the producers negotiate the introductory price, and the couple patient/doctor determines the consumption of drugs. All this is done under the legal rules that have been set by the legislator, in particular the reference pricing system. In the benchmark case, the legal rule is simply that the price is negotiated at the introductory stage, and then remains constant. ${ }^{6}$

In most European countries, individuals have a high insurance coverage. We assume in the paper that patients benefit from full insurance coverage. ${ }^{7}$ Nevertheless, we relax this assumption in Section 8.

To keep in line with the standard backward induction analysis, we first describe the downstream agents and activities. Then we give details on the innovation stage.

\subsection{The retail market}

We focus on the case of a single pathology that can be treated with one of several available drugs. At each instant in time there is a population of size 1 of patients to be treated. We take into account the patients' heterogeneity with respect to the treatments by assuming that the effect of a given medication varies among patients in terms of efficiency, tolerance and side effects. A therapeutic class is a set of drugs that can be used to treat the pathology, and such that none unambiguously dominates the others, i.e. drugs are horizontally differentiated. This does not mean that the drugs inside a given class are all perfectly substitutable, but that in the absence of any specific information on the patient, a doctor would be indifferent between prescribing one or the other drug in the class. In other terms, each one has an equal chance to be effective. After examination of the patient, the doctor reaches an objective diagnostic which allows him to know what is the most adapted treatment. ${ }^{8}$ We consider here a relation of perfect agency between the patient and the physician. They are therefore viewed as a single agent, the consumer. ${ }^{9}$

Concerning the drugs consumed, we consider both vertical and an horizontal differentiation. Vertical differentiation is defined by levels (therapeutic classes). In order to simplify as much as possible the analysis, we assume that there are two levels indexed by $j=C$ or $N$, designing respectively current and new, and that there

\footnotetext{
${ }^{6}$ The model is directly inspired by the French regime of administrated prices. Each new drug goes through three rounds before being introduced on the market: approval, evaluation of the therapeutic value of the drug, price decision. Each round is conducted by a separate body. The price of new drugs eligible for public insurance is fixed by the Comité économique des produits de santé (CEPS). According to CEPS the key elements are the improvement of medical service and the prices of drugs with similar therapeutic usage.

7 In France for example, patients are covered by the compulsory public insurance under a copayment rule, and most of them also benefit from complementary health insurance (only $8 \%$ of the people are not covered). The resulting effective rate of insurance is very high at the drug level. That makes the full reimbursement hypotheses acceptable.

8 This assumption is related to the concept of ex-post differentiation, as for instance in Gal-Or (1997, 1999).

9 With this assumption, we ignore two aspects with opposite effects: the so-called induced demand and the fact that physicians are incentivized by health authority and insurers to account for cost. In other words, doctors are assumed to be "bad agents" for the regulator. Relaxing these assumptions may also allow to leave some room for advertising, both informative (see Brekke and Kuhn, 2006) and persuasive (Hurwitz and Caves, 1988).
} 
are at most two drugs active in each level, $i=1$ or 2 . We adopt a discrete choice model to represent the utility from consumption. ${ }^{10}$ When consuming drug $i$ in level $j$, a patient obtains a gross utility that varies within the population, due to individual factors affecting efficiency and secondary effects. We denote by $V_{j}>0$ the mean utility from the treatment of the therapeutic class $j$, and capture the dispersion of side effects by a parameter $\theta$. Each patient receives $V_{j}+\theta \tilde{x}_{j}^{i}$, where $\tilde{x}_{j}^{i}$ is a random variable identically and independently distributed across consumers and drugs, with a uniform distribution on the interval $[-1 / 2,1 / 2]$. Therefore, the patient $i$ 's net utility is given by

$V_{j}+\theta \tilde{x}_{j}^{i}-p+R$

where $p$ is the price paid and $R$ is the reimbursement received. ${ }^{11}$ We can view the ex ante value of a medication $V_{j}$ as the reference value. Since doctors are able to identify the utility component $\tilde{x}_{j}^{i}$ the ex-post value is $V_{j}+\theta \tilde{x}_{j}^{i}$ which differs from the reference value by a random term $\theta \tilde{x}_{j}^{i}$.

Denote by $v=V_{N}-V_{C}$ the quality differential between classes. We assume that $v$ is substantially larger than $\theta$.

Assumption 1. $\theta \ll v$.

From the previous assumptions, we can derive the following properties of the demands for drugs under the regime where all drugs are $100 \%$ covered:

1. Only drugs in the highest level available are consumed. ${ }^{12}$

2. The total demand for drugs is equal to 1 .

3. The demand is evenly spread between all the drugs in the same level of the therapeutic class.

Drugs are produced at zero cost. Moreover, Assumption 1 implies that when a drug is introduced in level $N$, producers of level $C$ have no sales.

\subsection{Price negotiation}

When a new drug is accepted for introduction in the market, its price is set by a negotiation process between the regulator and the laboratory. The negotiation is depicted by the Generalized Nash Bargaining Solution, with arbitrary bargaining power, $\alpha>0$ for the regulator and $1-\alpha$ for the firm, where the status-quo corresponds to not introducing the new drug. ${ }^{13}$ This can be viewed as a bargaining process a la Rubinstein with different discount factors for the regulator and the firm. The negotiators only care about current consumers' surplus for the regulator and current profits for the firm. Assuming that the firm's negotiators are maximizing short run profit instead of the discounted flow of profits is admittedly a shortcoming, although we believe this is a good approximation to reality. We discuss the case where firms are far sighted in Section 8.

The negotiated price maximizes the function:

$[W(\text { with innovation })-W(\text { without innovation })]^{\alpha}$

$$
\times[\pi \text { (with innovation })-\pi(\text { without innovation })]^{1-\alpha}
$$

\footnotetext{
10 See Anderson et al. (1992) for a presentation of discrete choice models.

11 We do not include the payment for tax and insurance premium, that are independent of consumption (at the individual level).

12 In other words, consuming in class $N$ and incurring the highest disutility is better than consuming in class $C$ and incurring the smallest disutility.

13 The bargaining model developed in this paper has been developed after various interviews, especially with the CEPS's director and some personals of the French Protection Social Ministry.
}

where $W$ is the welfare as perceived by the regulator, and $\pi$ represents the instantaneous profit generated by the sales of the drug. It is worth noticing that the R\&D expenses are sunk for all agents at the time of marketing. Therefore, they are not taken into account in the bargaining process. Hereafter, we assume that if the negotiation fails, the firm has no way to market its innovation and $\pi$ (without innovation) $=0 .{ }^{14}$

We distinguish two situations. First, when there is no reference pricing, the introductory price is also the basis of reimbursement: for each drug, expenses are fully reimbursed to consumers up to this price. When a reference pricing system is used, each producer still negotiates a price with the regulator, but the reimbursement rule is changed. If there are two drugs in a therapeutic class, patients are fully reimbursed of expenses up to the minimal price in the class, while they bear the full cost above this level.

As an illustration, even if the French regulator has not adopted a reference pricing system for therapeutic classes, there is one that is used for some categories of drugs out of patent protection, the "tarif forfaitaire de remboursement". Under this system the "princeps" (the patented brand) and all its generics are reimbursed on the basis of the (administered) price of the generics. The princeps producer can then decide to lower its price, or to maintain it at its regulated level. In this paper, we are not interested by the generics, but we shall use this type regulation as our reference pricing system. ${ }^{15}$

\subsection{The RED sector}

The upstream part of the sector is composed of laboratories under the supervision of the regulator, whose role at this stage is limited to guarantee drug safety. There are $M$ (large enough) laboratories active in the research sector of the pharmaceutical industry for the given pathology. Each laboratory is identified with a single project. ${ }^{16}$ We assume that once a firm has innovated, it obtains a perfect patent of infinite duration. At that time, it exits the innovative sector to enter the downstream market where it becomes producer of the drug at some arbitrary small cost. ${ }^{17}$

The process of innovation is incremental. When a laboratory innovates, it can either discover a new product in the same level as existing products (here, this occurs when there is only one product on the market), or it can discover a product in the superior level. ${ }^{18}$ The former will be referred to as horizontal innovation and the new drug as a follower or a me-too. The latter is referred to as vertical innovation and the corresponding drug as a pioneer. When investing in $R E D$, the laboratory can devote resources to search for a superior quality drug, or for a drug within the existing therapeu-

\footnotetext{
14 It is worth noticing that our negotiation framework can also capture the bargaining process that occurs in United States between Pharmacy Benefit Managers (PBMs) and laboratories. PBMs are intermediaries that negotiate discounts and rebates with manufacturers and sign agreements with health plans. However, the discounts obtained by PBMs may also depend on quantity, which is not the case in our framework.

15 As pointed out by a referee, it is worth noticing that the second drug can be interpreted as a generic if $\theta$ captures differences in patients' attitude toward them. Nevertheless, implications in terms of R\&D and entry would be different.

16 The purpose of this assumption is to avoid the problem of the management of projects and products portfolio. It also reflects the opinion among specialists that innovation by independent research units is becoming standard in the pharmaceutical industry. Moreover, this assumption makes sense when considering a single pathology (see Section 9).

17 Another possibility is that the laboratory sells the patented innovation to a single producer. See Aghion and Tirole (1994) for opening the black box of innovation in an incomplete contracts framework.

18 We implicitly assume that ex ante, laboratories know what type of innovation they will obtain. In practice, there is much uncertainty on the final output of R\&D, but the distinction between major innovation and minor incremental innovation is key for the industry. See Berndt et al. (2006) or Dranove and Metzler (1994).
} 
tic class. If the current active level is $C$, a laboratory investing in the vertical dimension has thus access to level $N$. The pharmaceutical laboratories, being far sighted, correctly anticipate their future stream of income, and base their decisions on discounted profits, with a discount factor $r$.

In what follows, we assume that at date zero there is one drug in level $C$. We then distinguish two cases, one where follower innovation can occur only in level $N$, and one where it can occur in both levels, i.e. levels $C$ and $N$.

As regards the innovation process, ${ }^{19}$ we adopt a deterministic innovation model according to which laboratories choose the time devoted to develop the new product. This time is known in advance and the development cost is a decreasing function of the discovery time. By this, we mean that, ceteris paribus, a laboratory has to provide more costly efforts if it wants to reduce the duration of the investigation process. Formally, the cost of bringing a vertical innovation to the market is

$C(t)=C e^{-\gamma t}$

where $t$ is the time length of the research process. We consider the regular case where $\gamma>r$.

Investment cost is paid up-front so that the discounted profit of the firm investing at date $\tau$ is $e^{-r \tau}\left(-C(t)+e^{-r t} \Pi_{\tau+t}\right)$ where $\Pi_{\tau+t}$ is the expected discounted flow of profit obtained on the retail market following the introduction of the drug at date $\tau+t$.

Similarly, starting the process after the introduction of the first drug at a given level, the development of a new drug in the same level (horizontal innovation) has a cost

$c(t)=c e^{-\gamma t}$

with $c<C$. The discounted profit is then $e^{-r \tau}\left(-c(t)+e^{-r t} \Pi_{\tau+t}\right)$.

An investment plan thus consists in a date of investment $\tau$, a delay $t$ and the nature of innovation.

We consider a free-entry equilibrium in the innovation market where investment is publicly observable and a firm's objective is discounted profit. Therefore, an equilibrium consists in investment plans for each laboratory such that, when each laboratory anticipates correctly the plans of the other laboratories ${ }^{20}$ :

(i) no laboratory investing obtains a negative discounted profit;

(ii) there is no opportunity for a non-investing laboratory to enter with positive discounted profits.

At any time there are only two possible innovations (horizontal and vertical) and for each type, only the first to innovate enters. So condition (i) implies that at most one firm invests in a given type of innovation. Because a firm can always be the first to innovate by investing more than other investors, condition (ii) implies that active innovators obtain a zero discounted profit. Thus at a freeentry equilibrium three properties hold. First, the research projects start just after a new level is created. Second, the delay chosen for any active research project is the delay that cancels the profit of the investor. Third, if no laboratory invests in horizontal innovation, the profit from doing so is non-positive.

\footnotetext{
19 A description of the innovation process can be found in Dimasi et al. (1991) and Dimasi et al. (2003).

${ }^{20}$ It is worth noticing that the equilibrium features are in line with many other models of technological change (see for instance chapter 7 of Barro and Sala-iMartin, 1995).
}

\section{The retail market}

We denote by $i=1$ and 2 the first and the second drugs introduced in level $j$ and by $p_{j}^{1}$ and $p_{j}^{2}$ the price at which they are introduced.

When there is no reference pricing, consumers are fully reimbursed. Thus demand is price-inelastic. The optimal price for producers is then the administrated price that has been negotiated for their product. In particular the entry of a competing drug in the therapeutic class has no impact on the price of the existing drug, but it does affect the sales. With two drugs in the class, sales are evenly shared.

The reference pricing system matters for equilibrium prices only when there are two drugs in the same class $j$. Indeed, once the new drug is introduced the producer of the pre-existing drug may decide to maintain its price or reduce it. ${ }^{21}$ Let $p_{j}^{1}$ and $p_{j}^{2}$ be the regulated prices of the two drugs, and assume that $p_{j}^{1} \geq p_{j}^{2}$ (we show below that this is indeed the case). First, note that no price will be set below $p_{j}^{2}$, since below this level demand is price insensitive. Thus the only question is the choice of price by the producer of drug 1 . For any price $p$ between $p_{j}^{2}$ and $p_{j}^{1}$, its demand is $\operatorname{Prob}\left\{\tilde{x}_{j}^{1}-\tilde{x}_{j}^{2} \geq\right.$ $\left.\left(p-p_{j}^{2}\right) / \theta\right\}$ since patients pay $p-p_{j}^{2}$ to acquire the drug 1 . It is $1 / 2$ if it chooses to align its price to $p_{j}^{2}$.

Lemma 1. If $p_{j}^{1}>p_{j}^{2}>\theta / 2$, then under reference pricing the producer of the pioneer drug 1 aligns its price with the reference price $p_{j}^{2}$.

\section{Proof. See Appendix A.}

This strategy whereby laboratories choose to align their prices with the reference price is quite common ${ }^{22}$ even if, in practice, it may depend on reference pricing modalities. For instance, this result is in line with Danzon and Liu (1996) and Danzon and Ketchman (2004) who validate the "convergence hypothesis" to the reference price within a therapeutic class. As pointed out by LopezCasanovas and Puig-Junoy (2000), in Italy, laboratories "have now agreed to cut prices, to avoid delisting". These authors argue that in Sweden, drugs prices also drop to the reference pricing level. ${ }^{23}$

\subsection{Price negotiation without reference pricing}

The welfare function is given by the aggregate utility of consumers net of the social cost of health expenditures. With a single drug in the highest level available, all patients consume the same drug and we write welfare as

$W=V_{j}-p_{j}^{1}$.

This welfare function reflects the current concern for health expenditures, and summarizes the impact of budget considerations in the behavior of the regulator. In this objective, we assume no distortionary effect of taxation. We could account for a social cost $\lambda$ of public funds by using a welfare function $V_{j}-(1+\lambda) p_{j}^{1} \cdot{ }^{24}$ However, because consumers are fully reimbursed, this is formally equivalent in our model to renormalizing utility and prices by dividing them

\footnotetext{
21 We could also assume that the only choice is between the negotiated price and the reference price, which would strengthen our results.

22 See Brekke et al. (2007)

23 In practice, leading brands do not always choose to align. This could be explained by several factors as demand inertia, the fact that some countries determine prices through an external reference pricing mechanism, or the threat of parallel import. ${ }^{24}$ See Laffont and Tirole (1993) for a discussion of the concept of the social cost of public funds.
} 
by the factor $1+\lambda$. We thus interpret the utility and the prices as being normalized in unit of public funds. ${ }^{25}$

When there are two drugs in the highest level $j$, consumers are spread between the two drugs and the social value is the expectation of the highest value of treatment $V_{j}+\theta \max \left\{\tilde{x}_{j}^{1}, \tilde{x}_{j}^{2}\right\}$ minus the social cost. Using $1 / 6=E\left(\max \left\{\tilde{x}_{j}^{1}, \tilde{x}_{j}^{2}\right\}\right)$, we obtain

$W=V_{j}+\frac{\theta}{6}-\frac{1}{2}\left(p_{j}^{1}+p_{j}^{2}\right)$.

Introductory prices can be determined sequentially as the function of the introductory prices of previous drugs. The outcome of negotiation depends on the actual state of the markets (drugs on the markets and prices) and on the nature of the innovation (vertical or horizontal). There are three possible cases, when the current level is $j$.

Let us first consider the case of the introduction of a follower, in an existing level. There is a drug $i=1$ in the level $j$ at price $p_{j}^{1}$ (with $j=C$ or $j=N$ ), and the innovator introduces another drug $i=2$ in the same level. The new drug reaches half of the patients. The regulator's gain from the introduction at price $p_{j}^{2}$ of the new drug is $\theta / 6+\left(p_{j}^{1}-p_{j}^{2}\right) / 2$ where the first term represents the increase in therapeutic value, while the second captures the cost saving accounting for the fact that half of the population pays the new price. $^{26}$ The profit from introduction is $p_{j}^{2} / 2$. We thus solve the program

$\max _{p_{j}^{2}}\left(\frac{\theta}{6}+\frac{1}{2}\left(p_{j}^{1}-p_{j}^{2}\right)\right)^{\alpha}\left(\frac{p_{j}^{2}}{2}\right)^{1-\alpha}$

which yields a price

$p_{j}^{2}=(1-\alpha)\left[p_{j}^{1}+\frac{\theta}{3}\right]$.

We see that $p_{j}^{2}<p_{j}^{1}$ whenever $\theta$ is small compared to $p_{j}^{1}$. When the bargaining power of the regulator is high enough or the new drug has little value added, horizontal innovation leads to a reduction in health expenditure. In practice, the conditions of introduction of me-toos depend on the quality improvement provided by metoos. Nevertheless, various studies, such as the Arzneiverordnugs Report (2005) in Germany or DiMasi (2000) reveal that me-toos drugs usually imply high discounts relative to the price leader.

Consider now the introduction of a new drug at the superior level (pioneer). The new price depends on the prevailing situation on the market. Let $p_{C}^{a}$ be the average price in the level $C$, and $V_{C}+\theta_{C}^{a}$ be the average utility in level $C$ prior to the introduction of the new drug. Thus $\theta_{C}^{a}$ is the mean value of individual effects. When there is only one drug in level $C$, then $p_{C}^{a}=p_{C}^{1}$ and $\theta_{C}^{a}=0$, while with two drugs we have $p_{C}^{a}=\left(p_{C}^{1}+p_{C}^{2}\right) / 2$ and $\theta_{C}^{a}=\theta / 6$.

The gain in therapeutic value from introduction is $v-\theta_{C}^{a}$ since all patients switch to the new drug, which replaces the old one(s). So the negotiated price is the solution of

$\max _{p_{N}^{1}}\left(v-\theta_{C}^{a}-\left(p_{N}^{1}-p_{C}^{a}\right)\right)^{\alpha}\left(p_{N}^{1}\right)^{1-\alpha}$,

and the price of the new drug is

$p_{N}^{1}=(1-\alpha)\left[p_{C}^{a}+v-\theta_{C}^{a}\right]$

\footnotetext{
${ }^{25}$ Formally, if $\lambda>0$, the Lemma 1 should be modified to $p_{j}^{1}>p_{j}^{2}>\theta(1+\lambda) / 2$.

${ }^{26}$ Given that demand is not sensitive to price, there are no incentives for the seller of the existing drug to reduce its price when an entry occurs.
}

This deserves several comments. First, given that $v>\theta$, when there is only one drug in level $C$, the innovation to the superior level leads to an introductory price $p_{N}^{1}$ larger than the introductory price $p_{C}^{2}$ that would prevail for an horizontal innovation. Thus, a vertical innovation generates higher prices than an horizontal innovation. Second, since $p_{C}^{2}<p_{C}^{1}+\theta / 3$, the price of the pioneer is smaller when there has been a follower in the level $C$. There are indeed two effects at work. First, an horizontal innovation reduces health expenditures in the previous therapeutic class, forcing vertical innovators to negotiate a lower price. Second, the larger value in the level $C$ due to diversity reduces the gain in terms of therapeutic value brought on by the vertical innovation, again calling for lower prices.

Overall it is easy to verify that under Assumptions 1 and 2, the introduction of the pioneer raises the average price, while the introduction of a follower reduces it. Thus, for given delays of introduction, ceteris paribus, horizontal innovation reduces health expenditures, while vertical innovation raises them.

\subsection{Price negotiation under reference pricing}

We maintain the assumption on the bilateral bargaining process between the producer of the new drug and the regulator. Two points are worth noticing. Despite the fact that the producers of existing drugs are directly affected by the price negotiated for new drugs (since their drug reimbursement level is affected), these producers are not part of the negotiation process. Moreover, the bargaining weights $\alpha$ and $1-\alpha$, are not affected by the change in the bargaining environment.

In this case, we denote by $P_{j}^{i}$ the negotiated prices instead of $p_{j}^{i}$ (prices without reference pricing). First, notice that the behavior of negotiators when the level $N$ pioneer drug is introduced remains unchanged, compared to before, since reference pricing is irrelevant when there is a single drug within the class: $P_{N}^{1}=$ $(1-\alpha)\left[P_{C}^{a}+v-\theta_{C}^{a}\right]$. The main difference with the base regime is when a new drug is introduced in an existing level (follower). In this case, the regulator anticipates that negotiating an introductory price with the follower that is below the price of the pioneer will induce the producer of the latter to align its price, as shown in Lemma 1. Thus the solution of the bargaining game is given by

$\max _{P_{j}^{2} \leq P_{j}^{1}}\left(\frac{1}{6} \theta+P_{j}^{1}-P_{j}^{2}\right)^{\alpha}\left(\frac{P_{j}^{2}}{2}\right)^{1-\alpha}$,

which yields the price

$P_{j}^{2}=(1-\alpha)\left(P_{j}^{1}+\frac{\theta}{6}\right)$.

The corresponding price $P_{j}^{2}$ is strictly smaller than $P_{j}^{1}$ when $P_{j}^{1}>$ $\theta(1-\alpha) / 6 \alpha$. Comparing with above we obtain that:

Lemma 2. For a given introductory price of the pioneer, a follower is introduced at a smaller price with reference pricing than without.

Proof. Follows from $P_{j}^{2}<(1-\alpha)\left[P_{j}^{1}+\theta / 3\right]$.

Results of Lemma 2 leads to the following remark:

Remark 1. Under reference pricing, the regulator is a tougher negotiator.

The reason is that the marginal benefit for the regulator of reducing the price is higher because the price reduction applies to all the drugs in the class. All other things equal, the reference pricing regulation increases the regulator's bargaining position and leads to lower prices. 


\section{The dynamics of innovation within a class}

In all that follows we assume that at date zero there is one drug in level $C$ and we take $p_{C}^{1}$ as given by history: $p_{C}^{1}=P$. For consistency, we impose two conditions on $P$ :

Assumption 2. $\theta \ll P<\frac{1-\alpha}{\alpha} v$.

The first condition follows from Assumption 1 with the same interpretation. The second comes from the presumption that $P$ is the result of past innovation. More precisely, it constitutes a starting value for the price that can be negotiated for sequence of pioneer innovations of incremental values $v$. Notice that the assumption implies that the condition of Lemma 1 holds.

We first consider the impact of reference pricing when innovation is not possible in level $C$ (no me-too in level $C$ ). This allows us to focus on the impact of the regulatory regime on the creation and the within dynamics of a new therapeutic class, here the level $N$. We leave to Section 5 the analysis of the long run impact of the regulatory regimes, accounting for the introduction of a me-too in level $C$.

\subsection{The dynamics of innovation without reference pricing}

Knowing the prices that will be set at the marketing stages, we can now solve the innovation game. Since the cost of a vertical innovation goes to zero asymptotically, in any equilibrium, all levels will be discovered at some points in time. Moreover introductory prices have been determined above. Thus, when looking at the dynamics of innovation, the questions concern the delays before the occurrence of the pioneer and the follower innovations in level $N$. Remind that concerning delays, the "clock" is set to zero once the pioneer drug is introduced.

In the absence of reference pricing, the introductory prices will be $p_{N}^{1}$ and $p_{N}^{2}$ given by the price Eqs. (1) and (2), with $p_{C}^{a}=P$ and $\theta_{C}^{a}=0$. The pioneer is introduced after a delay $t_{N}$ and the follower after an extra delay $\tau_{N}$, thus at $t_{N}+\tau_{N}$. We assume the following conditions that ensure that the drugs will be introduced after some delays, $\tau_{N}>0, t_{N}>0$ :

Assumption 3. $C>\frac{(1-\alpha)(P+v)}{2 r}$ and $C>\frac{(1-\alpha)(P+v)}{r}$.

Let us now consider innovation delays by backward induction. When the pioneer is introduced, the research focuses on the follower with an investment such that the discounted benefits are null, that is the delay $\tau_{N}$ from introducing the second drug is solution of $c e^{-\gamma \tau_{N}}=\left(e^{-r \tau_{N}} p_{N}^{2}\right) / 2 r$, which yields an introductory delay

$\tau_{N}=\frac{1}{\gamma-r}\left(\ln (c)-\ln \left(\frac{p_{N}^{2}}{2 r}\right)\right)$.

Consider now the pioneer. In equilibrium, a pioneer enjoys monopoly sales for a period of length $\tau_{N}$ following its introduction, and serves half of the market afterward. The expected profit is thus $p_{N}^{1} / r\left(1-\left(e^{-r \tau_{N}}\right) / 2\right)$. The free-entry zero profit condition then implies that the laboratory providing a pioneer chooses a delay $t_{N}$ solution of

$C e^{-\gamma t_{N}}=e^{-r t_{N}} \frac{p_{N}^{1}}{r}\left(1-\frac{e^{-r \tau_{N}}}{2}\right)$

Using Eqs. (4) and (1), we obtain

$t_{N}=\frac{1}{\gamma-r}\left(\ln C-\ln \left[\frac{\chi\left(p_{N}^{1}\right)}{r}\right]\right)$, where the function $\chi$ is defined as

$\chi(p)=p-\left(\frac{(1-\alpha)[p+(\theta / 3)]}{2 c r}\right)^{r /(\gamma-r)} \frac{p}{2}$.

This last function summarizes the effects of the introductory price on the pioneer's profitability, accounting for the effect on the me-too introductory delay. Preserving zero discounted innovation profits requires that an increase in profitability of a project is compensated with a shorter delay, which increases research costs. Thus there is a negative relationship between $\chi$ and $t_{N}$.

Reducing the price depresses the margin obtained by the producers, but it also reduces the price of the me-too. This reduction of the me-too's price delays its introduction, thus increases the length of the period during which the innovator is a monopoly. The overall effect on profits is ambiguous. Nevertheless we obtain:

Lemma 3. If $\gamma>2 r$, then $\chi(p)$ is increasing.

Proof. See Appendix A.

In other words, the direct effect of the price on the profit margin dominates if the innovation cost declines fast enough.

\subsection{Innovation under a reference pricing regulation}

Let us now consider the case with reference pricing. The difference with the previous case is twofold. First, negotiated prices are different. Second, the pioneer reduces and aligns its price at the introduction of the follower ( $c f$. Lemma 1 ).

Introducing reference pricing under our assumption does not change the price of the pioneer: $P_{N}^{1}=p_{N}^{1}$ as we consider in this section level $C$ drug prices as exogenous and identical under both regulatory regimes. On the contrary, for the follower the price changes to (using the price Eq. (3)):

$P_{N}^{2}=(1-\alpha)\left[P_{N}^{1}+\frac{\theta}{6}\right]=p_{N}^{2}-(1-\alpha) \frac{\theta}{6}$.

Under reference pricing, denote $\varsigma_{N}$ the introductory delay of the follower and $T_{N}$ the delay of introduction of the pioneer. The delay for the follower in the level $N$ is given by

$\varsigma_{N}=\frac{1}{\gamma-r}\left(\ln (c)-\ln \left(\frac{P_{N}^{2}}{2 r}\right)\right)$.

The introductory delay of the pioneer is now given (with similar notations as above) by

$T_{N}=\frac{1}{\gamma-r}\left(\ln (C)-\ln \left[\frac{\Xi\left(P_{N}^{1}\right)}{r}\right]\right)$

where

$\Xi(p)=\left(1-e^{-r \zeta_{N}}\right) p+\frac{1}{2} e^{-r \varsigma_{N}}(1-\alpha)\left[p+\frac{\theta}{6}\right]$.

$\Xi(p)$ represents the average flow profit for the pioneer under reference pricing accounting for the new delay of introduction and the new price of the follower.

Lemma 4. If $(1-\alpha) \gamma>2 r$, then $\Xi(p)$ is increasing.

Proof. See Appendix A.

This last function is similar to $\chi($.$) in the regime without refer-$ ence pricing and is detailed in the Appendix A. The difference is that after delay $\varsigma_{N}$ the pioneer not only looses half of the market but its price is reduced to $P_{N}^{2}$. For this reason, the condition to ensure that $\Xi(p)$ increases in $p$ is more restrictive than for $\chi($.$) and depends on$ 
the regulator's bargaining power: if $\alpha$ is low enough, the profitability of the pioneer increases with its price. The effect of the duration of the monopoly period is stronger because, with reference pricing, the pioneer is forced to reduce its profit margin at the introduction of the me-too (Lemma 1).

Proposition 1. In terms of delays of introduction, the consequences of the reference pricing are:

(i) The me-too is delayed i.e. $\varsigma_{N}>\tau_{N}$.

(ii) The pioneer is delayed i.e. $T_{N}>t_{N}$ if and only if $P$ is above some threshold.

Proof. See Appendix A.

Under the reference pricing regime, the regulator sets lower prices for the me-too and the introduction of me-toos is delayed compared to the case without reference pricing. The effect on the innovation delay of the pioneer is more ambiguous. Indeed, adopting reference pricing generates two conflicting effects on the profitability of the pioneer. For one thing, the pioneer drug benefits from a longer period of monopoly. But when the me-too is introduced, it supports a larger profit loss as its price is aligned.

Notice that the relationship between $P$ and the introductory price of the pioneer is not affected by the regulation regime, i.e. $P_{N}^{1}=p_{N}^{1}$. Because the marginal effect of the introductory price on profit is stronger under no reference pricing than under a reference pricing regulation, the profitability of the pioneer introduction is higher under no reference pricing for high values of $p_{N}^{1}$, thus for high values of $P$.

Corollary 1. It is more likely that reference pricing delays pioneer innovation if the incremental value $v$ is large, the incremental value of the me-too is small ( $\theta$ small), the firms are patient ( $r$ small). The effect of the bargaining power $\alpha$ is ambiguous.

Proof. See Appendix A.

The condition on $v$ resumes exactly the same intuition as the condition on $P$ in Proposition 1. If $v$ is high enough, ceteris paribus, the negotiated price of the pioneer in level $N$ will be high too, generating a higher profitability under no reference pricing than under reference pricing. Moreover, under a reference pricing regime, when the incremental value of the me-too is small, the follower negotiates a low price with the regulator. This low price being applied to both drugs in the class, it decreases the profitability of the pioneer when it looses its monopoly position. This reduction of the pioneer's profitability delays its introduction in comparison with no reference pricing. Notice that for small differentiation $(\theta$ small), both the pioneer and the follower innovations are delayed by the introduction of reference pricing.

\section{The long run effect of reference pricing}

In Section 4, the level $C$ was taken exogenously and we could focus on the impact of the regulatory regime on the level N's characteristics, i.e. levels of prices and delays of introduction. Nevertheless, the choice of the regulatory regime generates more complex effects since it influences innovation in the current class as well as in future classes. To study this issue we now allow innovation to occur in level $C$ (prior to the discovery of the first level $N$ drug). The new question is thus whether or not there is an horizontal innovation in level $C$. This leads to distinguish two types of equilibria, corresponding to two different sequences of innovation.

In the first possible sequence of innovations, the follower in level $C$ is never introduced. We refer to such a situation as involving a "short sequence" as less drugs appear and the second level appears faster than in the second type of equilibrium. In the second possible type of equilibrium, the follower of level $C$ is introduced before the pioneer of level $C$. We refer to this as involving a "long sequence".

\subsection{Innovation without reference pricing}

In what follows we use symbol $k \in\{s, l\}$ to denote the two types of equilibrium, where $s$ and $l$ stand respectively for short and for long. We denote $\tau_{C}(k)$ and $\tau_{N}(k)$ the delay of introduction of the follower in the sequence $k$ in levels $C$ and $N$, when this introduction occurs in equilibrium, and $t_{N}(k)$ the delay of apparition of the level $N$ pioneer. Finally $p_{j}^{i}(k)$ represents the introductory price of the drug $i$ in level $j$ corresponding to a sequence $k$. Notice that in level $C$, the research on the follower and the research on the pioneer both start at date zero. In other words, the firm planning to introduce the next pioneer benefits from starting the research process immediately instead of waiting for the follower to emerge. Indeed, according to the fact that the follower does not bring any incremental value, this minimizes the introductory delay. Thus the follower is introduced at date $\tau_{C}(l)$ in the long sequence, the next level at date $t_{N}(k)$ and the last drug (the me-too in the level $j=N$ ) at date $t_{N}(k)+\tau_{N}(k)$.

For any sequence, the introductory prices are independent of introductory dates, but it is worth noticing that the introductory price of the pioneer depends on the sequence of innovations:

$p_{N}^{1}(s)=(1-\alpha)[P+v]$,

$p_{N}^{1}(l)=(1-\alpha)\left[\frac{P+p_{C}^{2}(l)}{2}+v-\frac{\theta}{6}\right]$.

To characterize equilibria, we follow the same reasoning as in the previous section. We provide the formal derivation in the Appendix A and summarize here the intuitions and the results.

For each sequence, we can compute the equilibrium choices of introductory delays $t_{N}(k)$ and $\tau_{N}(k)$ using Eqs. (5) and (4) for respective prices $p_{N}^{1}(k)$ and $p_{N}^{2}(k)$. The question that remains is whether the equilibrium is of type $k=s$ or of type $k=l$. The answer depends obviously on the incentives to introduce a follower in level $C$. Notice that the profit from introducing a follower in level $C$ when the pioneer is planned to arrive at $t_{N}$ is ${ }^{27}$ :

$\max _{\tau \leq t_{N}}\left\{\frac{p_{C}^{2}(l)}{2 r}\left(e^{-r \tau}-e^{-r t_{N}}\right)-c e^{-\gamma \tau}\right\}$,

which increases with the delay $t_{N}$. This value of innovation in level $C$ is positive if the pioneer is introduced after some critical lapse of time $\bar{t}$. Thus it is profitable to introduce a follower in level $C$ if and only if $t_{N} \geq \bar{t}$. It follows that an equilibrium with a short sequence exists if and only if $t_{N}(s) \leq \bar{t}$. Indeed, only in this case, no innovator will choose to invest in a follower in level $C$.

Similarly an equilibrium with a long sequence exists if and only if $t_{N}(l) \geq \bar{t}$. In this case we can then compute the introductory delay $\tau_{C}(l)<t_{N}(l)$ which cancels the profit.

To characterize equilibria, we need to compare $t_{N}(s), t_{N}(l)$ and $\bar{t}$. Given that $p_{N}^{1}(s)>p_{N}^{1}(l)$, the pioneer emerges faster when its introductory price increases if $\chi(p)$ is increasing in $p$ (Lemma 3). We can then conclude:

Proposition 2. There exists a threshold $\bar{t}$ such that an equilibrium with a short sequence exists if and only if $t_{N}(s) \leq \bar{t}$, and an equilibrium with a long sequence exists if and only if $t_{N}(l) \geq \bar{t}$. For $\gamma>2 r, t_{N}(s)<$ $t_{N}(l)$ and an equilibrium exists.

\footnotetext{
27 An innovation would allow to sell $1 / 2$ at price $p_{C}^{2}(l)$ as long as the pioneer in level $N$ is not introduced. Note that the price corresponds to the long sequence as it is conditioned on the entry of the follower in level $C$.
} 


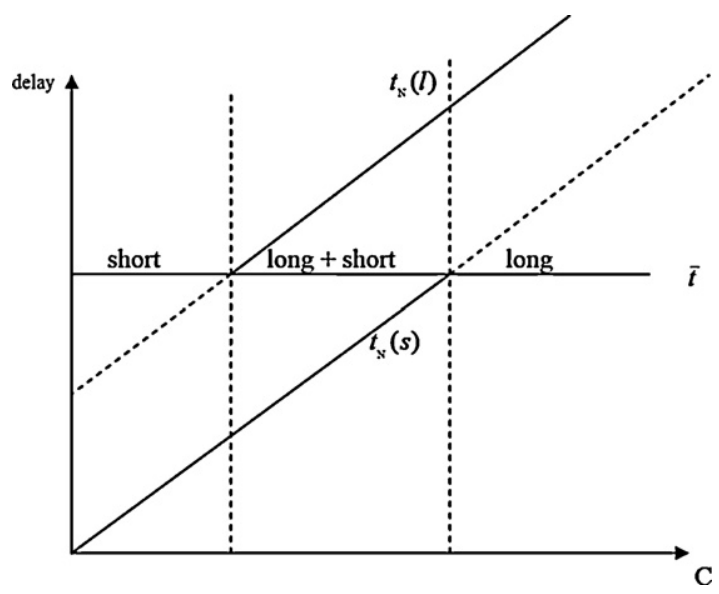

Fig. 1. Introductory delays.

Proof. See Appendix A.

Notice that in the case where $t_{N}(s) \leq \bar{t} \leq t_{N}(l)$, both sequences can emerge in equilibrium. The situation where $\gamma>2 r$ is illustrated in Fig. 1 which represents the threshold $\bar{t}$ and the delays $t_{N}(s)$ and $t_{N}(l)$ as a function of the parameter $C$. When the pioneer innovation is not costly, only the short sequence emerges, while for high $C$ only the long sequence occurs. For intermediate values the two sequences can emerge as equilibria.

Considering the impact of various parameters on the type of equilibrium we find that:

Corollary 2. For both sequences $s$ and $l$, the sequence short is less likely $\left(\bar{t}-t_{N}(k)\right.$ decreases) when $C$ increases or $\theta$ increases. The same is true when $v$ decreases if $\gamma>2 r$.

Proof. See Appendix A.

As expected, increasing the value of vertical innovations also increases the likelihood to see such innovations on the market. Intuitively, increasing the innovative content $v$ of vertical innovations should tend to favor a short sequence, with less me-too introductions. The reason is that me-toos have shorter prospect for existence. However, it is worth noticing that the opposite counterintuitive effect could arise if $\chi(p)$ is decreasing on some range.

The impact of the bargaining power on the dynamics is more complex. Increasing $\alpha$ reduces the prices and delays the follower in level $N$. In sequence $s$, the result is to accelerate the introduction of the pioneer. However, when considering a long sequence, the relative price $p_{N}^{1}(l) / p_{C}^{2}(l)$ decreases, which works in opposite direction.

\subsection{Innovation under a reference pricing regulation}

Introducing reference pricing changes the price of the level $N$ me-too to

$P_{N}^{2}(k)=(1-\alpha)\left[P_{N}^{1}(k)+\frac{\theta}{6}\right]$.

The price of the pioneer in each sequence is

$P_{N}^{1}(s)=p_{N}^{1}(s)$ and $P_{N}^{1}(l)=p_{N}^{1}(l)-\frac{\alpha(1-\alpha)}{2} P$

while the level $C$ me-too is introduced at price $P_{C}^{2}(l)=p_{C}^{2}(l)-(1-$ $\alpha) \theta / 6$. Thus we obtain:

Proposition 3. For a given sequence of innovations, all the prices are smaller with the reference pricing system than without it.
Proof. Immediate for the level $C$ me-too and the pioneer. For the level $N$ me-too, this follows from the fact that (3) implies lower prices than (1).

Given the new prices, the derivation of the equilibria is the same as in the case with no reference pricing except that the introductory delays $\varsigma_{N}(k)$ and $T_{N}(k)$ are given by Eqs. (6) and (7) applied to the relevant price. The effect of reference pricing on the pioneer's profits is ambiguous due to the fact that the introduction of the follower is delayed, and thus the period of monopoly sales for the pioneer increases. However, direct computation shows that $t_{N}(k)<T_{N}(k)$ if $\theta$ is small.

Again, which equilibrium emerges depends on the relative position of the pioneer introductory delay with respect to a threshold.

Proposition 4. There exists a threshold $\bar{T}>\bar{t}$, such that an equilibrium with a short sequence exists if and only if $T_{N}(s) \leq \bar{T}$, and an equilibrium with a long sequence exists if $T_{N}(l) \geq \bar{T}$. For c not too small, or for $\gamma>2 r /(1-\alpha), T_{N}(s)<T_{N}(l)$ and an equilibrium exists.

Proof. See Appendix A.

We see that the conclusions are the same as for Proposition 2, except that the thresholds take different values. It is worth noticing that $\bar{T}>\bar{t}$ suggesting that reference pricing may facilitate the emergence of a short sequence. In particular, this is the case if the profit of a pioneer in a short sequence is larger under reference pricing than under no reference pricing:

Corollary 3. If $\Xi\left(p_{N}^{1}(s)\right) \geq \chi\left(p_{N}^{1}(s)\right)$, then the short sequence is an equilibrium with reference pricing if it is without.

Proof. Follows from $\bar{T}>\bar{t}$ and $T_{N}(s) \leq t_{N}(s)$ since $P_{N}^{1}(s)=$ $p_{N}^{1}(s)$.

A direct computation shows that the profit of the pioneer in the short sequence is larger with reference pricing than without it if

$\frac{\left(p_{N}^{1}(s)+(\theta / 6)\right)(1+\alpha)-(\theta / 3)}{p_{N}^{1}(s)} \leq\left(\frac{p_{N}^{1}(s)+(\theta / 3)}{p_{N}^{1}(s)+(\theta / 6)}\right)^{r /(\gamma-r)}$

which occurs for some parameter values. In this case, the increase in the introductory delay of the me-too is large enough to outweigh the pioneer from the fact that this introduction will force him to align its price.

Nevertheless for other cases, the reverse condition holds, the profit of the pioneer decreases, $T_{N}(s)>t_{N}(s)$ and the impact is $a$ priori ambiguous.

For the purpose of illustration, consider the case where $\theta$ is close to zero. ${ }^{28}$ In this case, it is straightforward to see that condition (8) is violated. However, working directly with equilibrium conditions, we prove in the Appendix A that the impact of reference pricing remains stronger for the me-too innovations than for the pioneer, favouring short sequences.

Corollary 4. Suppose that $\theta$ is close to zero. The profit of a pioneer is smaller under reference pricing in both sequences. But if $2 / 3>$ $e^{-r \tau_{N}(s)}$, then the short sequence is an equilibrium with reference pricing if it is without.

Proof. See Appendix A.

To summarize, when the effect on the profit of a pioneer is not too strong, either because the monopoly period increases, or because this period is long, in any case, the dominant effect of a reference pricing system is the reduction in the introductory price

\footnotetext{
28 Obviously, $\theta$ that tends to 0 remains a hypothetical case. Indeed, as pointed out by an anonymous referee, there is a natural lower limit to $\theta$. If the differentiation is too low, it would make sense that the laboratory doesn't obtain a patent.
} 
of the me-toos. In that case, the reference pricing system tends to crowd-out the horizontal innovation.

\section{Welfare and health expenditures}

The consumers' welfare is computed using the same discount factor $r$ as the laboratories. If $U_{t}$ is the instantaneous consumers' surplus at date $t$, net of transfers, the intertemporal surplus is defined as

$r \int_{0}^{\infty} U_{t} e^{-r t} \mathrm{~d} t$.

Due to competition in the R\&D sector, there is no profit in any equilibrium, so that the total surplus coincides with the total consumer surplus. This surplus is the difference between the intertemporal utility from treatment and the discounted social cost of treatment. If there were no innovation, the intertemporal welfare would be $V_{C}-P$.

Let $w$ be the welfare gain in sequence $k$ compared to this benchmark level $V_{C}-P$. We can again decompose this gain in two terms: the first one is related to the gain in health, whereas the second one captures the variation in health expenditures. More precisely, if $k$ is the equilibrium sequence, we have $w=h(k)-e(k)$, where $h(k)$ is the aggregate gain in health in sequence $k$ and $e(k)$ is the increase in discounted health expenditures. The details of the computations and formulas are given in the Appendix A. For the case where the regulator uses a reference pricing regulation, and the equilibrium sequence is $k^{\prime}$, we have similarly $W=H\left(k^{\prime}\right)-E\left(k^{\prime}\right)$, where $H\left(k^{\prime}\right)$ and $E\left(k^{\prime}\right)$ have the same interpretations. It is worth noticing that expenses are modified to account for the alignment of the pioneer's price with the me-too's price.

The introduction by the legislator of a reference pricing regulation has several potential effects.

First, instantaneous health expenditures are affected through two channels:

- The new price applies to all drugs when an innovation occurs;

- The regulator negotiates different prices.

Second, the dynamics of innovation is affected in two possible ways:

- Within a sequence, introductory delays change;

- The equilibrium sequence may change, for instance the follower in level $C$ may be introduced without reference pricing but not with reference pricing.

When the legislator changes the regulatory regime to reference pricing, health expenditures are reduced for a fixed innovation path, but since the innovation path changes, the final impact depends on the effect on innovation. The changes in the pattern of innovation affects both the health level and the expenses.

It is worth noticing that there may be countervailing effects on expenses due to different delays. As seen above, under reasonable assumptions, horizontal innovations reduces total expenses. Thus, a larger delay in the introduction of followers would be detrimental to expenses, as well as the non-introduction of some me-toos. The results that precede suggest that reference pricing may generate such effects, since it raises delays for the follower in level $N$, and for a given date of introduction of the level $N$ pioneer, it also raises the delay for the follower in level $C$.

The introductory time of the pioneer in level $N$ is also affected in a non-trivial way. Indeed, two countervailing effects are involved. On one hand, the profitability of a pioneer is reduced because its price decreases when the follower is introduced. On the other hand, the delay of introduction of the follower increases, allow- ing the producer of a pioneer to benefit from a longer period of monopoly situation. The global effect of imposing reference pricing is thus ambiguous, even when there is no change in the innovation sequence.

The impact on health is also ambiguous. For a given sequence, one can expect reference pricing to have a negative impact on health by delaying innovations. But moving from a long sequence to a short sequence results in an ambiguous effect as there is no introduction of a me-too in level $C$ while the pioneer arrives after a smaller length of time.

\section{Numerical results}

In order to obtain more insights on the impact of the change of regime on the dynamics of innovation and on consumers welfare, we develop some numerical simulations. We use the market for statins in France as a way to benchmark some parameters of the model and to get insights on the likely impact of reference pricing on the dynamics of this type of drugs. ${ }^{29}$

\subsection{The statins}

Statins are the most effective drugs to reduce the LDL - cholesterol in blood. In France, the market for statins was born in 1989 with the introduction of Simvastatin $(20 \mathrm{mg})$. In the following 15 years, new products have been regularly introduced. In 2004 there were five available substances, with Simvastatin, Pravastatin (introduced in 1991), Fluvastatin (introduced in 1996), Atorvastatin (introduce in 1998) and Rosuvastatin (introduced in 2004). The first generic (for Simvastatin) appeared in year 2005, and should be followed by another one (for Pravastatin) in year 2006. Each substance is now available in 2 to 4 dosages.

These molecules have different performance levels, in particular in term of percentage reduction achieved for a given daily dose. ${ }^{30}$ They also differ on the nature of the secondary effect generated by each molecule, but we do not have data on this aspect. For a daily dose of $20 \mathrm{mg}$, the mean percentage reduction in "bad" cholesterol is 32\% for Simvastatin, 24\% for Pravastatin, 21\% for Fluvastatin and $43 \%$ for Atorvastatin. The last drug, Rosuvastatin generates a reduction of $48 \%$. Differences are significant at a $95 \%$ confidence interval, except between Pravastatin and Fluvastatin, and between Atorvastatin and Rosuvastatin. Similar patterns occur for higher doses ( $40 \mathrm{mg}$ or $80 \mathrm{mg}$ ).

This shows a clear difference between the first three molecules and the last two. Indeed this difference has allowed the producers of Atorvastatin and Rosuvastatin to launch products at daily doses of $10 \mathrm{mg}$, while the others stay above $20 \mathrm{mg}$. The corresponding reductions in cholesterol are $37 \%$ and $43 \%$, above the level achieved by the others at $20 \mathrm{mg}$.

The expansion in the supply came along with a very rapid growth of statin consumption. In economic terms, if we add all products together, the sales value grew at more than $17 \%$ per year over the period, despite regular price reductions. The average growth rate during the last 4 years, is still at $15 \%$. Even the oldest drug (Simvastatin $20 \mathrm{mg}$ ) continues being sold in growing quantities, despite the fact that it now suffers from the competition of other statins on the market. In fact there has been an extraordinary growth in the demand for statins, which has benefited all statins, and which is also the source

\footnotetext{
${ }^{29}$ We thank Pfizer France and Jean-François Guichard for providing the data used thereafter.

30 Percentage reduction are derived from Etude, BMJ 2003.326.7404.1423 by MR Law, NJ Wald et AR Rudnicka, 'Quantifying effect of statins on LDL cholesterol, ischaemic heart disease and stroke: systematic review and meta-analysis'.
} 


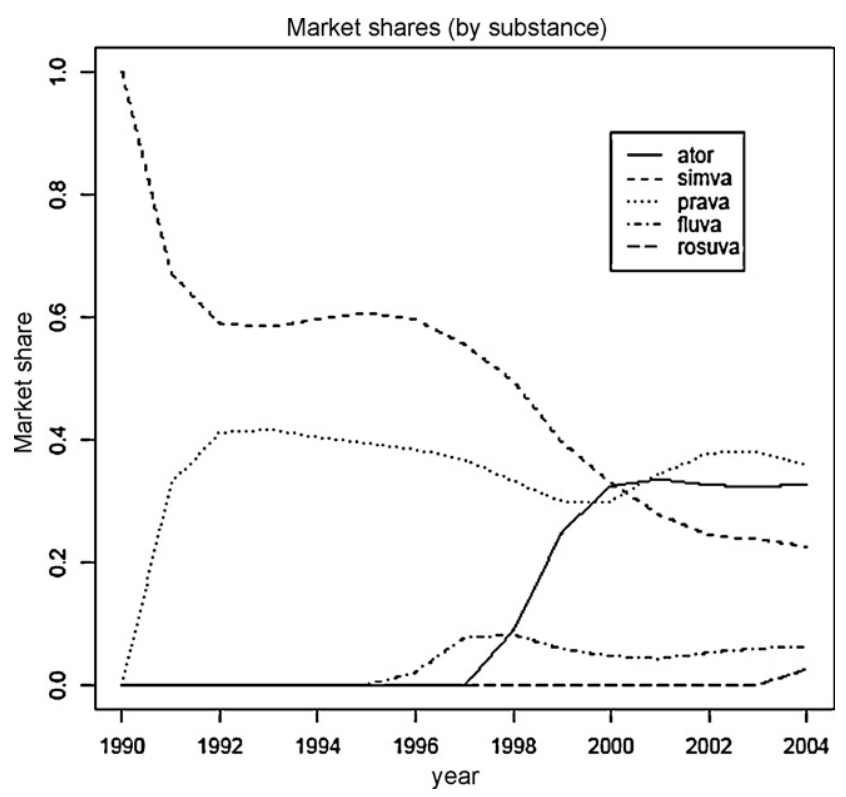

Fig. 2. Market shares for statins.

of an increase of expenditure for the health care system in France.

In Fig. 2, we depict the evolutions of market shares of each molecule. The market shares have dramatically changed over time. Simvastatin, the first statin to be introduced, remained leader until 2000 where it lost the first position to Pravastatin and Atorvastatin. Fluvastatin has expanded very little, and Rosuvastatin still has a very short history. For most substances there is a dosage that appears much more popular than the others and that sells about twice as much as the second dosage of the same substance.

Prices decreased along all the period. Simvastatin $20 \mathrm{mg}$ which was first sold at 30.05 Euros is sold at 19.34 at the end of the period. ${ }^{31}$ The other drugs experimented similar decline (see the table in Fig. 3). A particularity of the market, is that entrants were marketed at prices that were lower than those of older drugs, even if they were recognized to have a greater efficiency. This is the case for example of Atorvastatin $10 \mathrm{mg}$ which was first sold at 20.62, and was then therefore cheaper than the oldest Pravastatin and Simvastatin, although it is known for generating a greater decrease in cholesterol.

\subsection{Simulations}

We use the above data to get some insights on relevant parameters. First, as pointed above Fluvastatin had only a marginal impact on the market, while Rosuvastatin has just emerged. We thus decided to focus on the three main molecules, Simvastatin, Pravastatin and Atorvastatin. In each molecule some dosages seem to be determinant, in particular $20 \mathrm{mg}$ doses for the first two are largely dominant. From the above data, we decided to include the first two molecules in one level (in our model it corresponds to level $C$ ) and to classify Atorvastatin as a vertical innovation (level $N$ ), as defined in our model.

Our model has little to say about volumes and in any case demand has been increasing over time. ${ }^{32}$ So we use prices as a benchmark. Nevertheless, we face a difficulty because molecules

\footnotetext{
31 Prices are for a 4-week treatment.

32 Notice that our model can easily accommodate a time trend in demand by adjusting the discount factor. Indeed, increasing demand is formally equivalent to increasing $\delta$.
}

are available at different doses and we do not know which is the most relevant. We chose to compare Simvastatin $20 \mathrm{mg}$ with Atorvastatin $20 \mathrm{mg}$, as they have the same dose of statin.

The second issue relates to the fact that we observe a general trend in decreasing prices that is not accounted for in the model. This means that we cannot directly compare introductory prices. To avoid the issue, we choose the prices of a given year as a benchmark, namely 2004.

The data thus corresponds to a long sequence with an horizontal innovation in level $C$. We have 3 prices available namely 20.75 for Simvastatin, 18.92 for Pravastatin and 32.76 for Atorvastatin. Prices allow us to calibrate the equation of bargaining between the regulators and the innovator. However for prices, three parameters matter $\alpha, v, \theta$. We thus calibrated the last two as a function of $\alpha$. We present the predicted prices for each possible trajectories for $\alpha=0.2$. Patterns are similar for other values:

\begin{tabular}{lcll}
\hline$\alpha=0.2$ & $p_{C}^{2}$ & $p_{N}^{1}$ & $p_{N}^{2}$ \\
\hline no RP & 18.92 & 32.76 & 28.52 \\
RPseq s & - & 34.65 & 28.9 \\
RPseq 1 & 17.76 & 31.10 & 26.04 \\
\hline
\end{tabular}

Notice that prices are higher under a short sequence, due to the disappearance of the first innovation.

Delays are more difficult to interpret since in the model we ignore the intrinsic randomness of innovation. In the data we have a delay of 9 years for the vertical innovation, and 2 and 6 years for an horizontal innovation, so an average of 3 years. In our model $\hat{\tau}_{N}<\hat{\tau}_{C}$ which is not the case in the data. This may be due to randomness and/or to external effects such as the deflationary trend. We set $\gamma=0.5$ and we do not calibrate the costs parameters $c$ and $C$ but rather check that predicted innovation delays are consistent with observed patterns. For various values of $\alpha$ we adjust these costs to generate various scenarios. All our results have pointed to the same conclusions.

We present below the predicted trajectories for various scenarios. We also computed the discounted consumers' surplus (the term $h$ or $H$ in the surplus) and the discounted expenses (the term $e$ or $E$ ). More precisely, we computed the change in the benefits for consumers and in expenses compared to a situation with no innovation at all.

We summarize the results below

\begin{tabular}{llllll}
\hline$\alpha=0.2$ & $\tau_{C}$ or $\zeta C$ & $t_{N}$ or $T_{N}$ & $\tau_{N}$ or $\varsigma_{N}$ & Health & Expenses \\
\hline no RP & 4.8 & 11.4 & 0.73 & 20.11 & 6.13 \\
RP & 4.96 & 11.5 & 0.93 & 20.02 & 4.71 \\
no RP & 7.14 & 9.64 & 1.13 & 21.31 & 6.73 \\
RP & NO & 9.52 & 1.1 & 21.28 & 7.61 \\
& & & & & \\
$\alpha=0.4$ & $\tau_{C}$ or $\zeta C$ & $t_{N}$ or $T_{N}$ & $\tau_{N}$ or $\varsigma_{N}$ & Health & Expenses \\
\hline no RP & 5.1 & 10.76 & 0.93 & 39.49 & 5.58 \\
RP & 5.65 & 10.9 & 1.36 & 39.1 & 3.06 \\
no RP & 7.06 & 10.3 & 1.78 & 39.54 & 5.82 \\
RP & NO & 10.06 & 1.86 & 39.26 & 7.03 \\
& & & & & \\
$\alpha=0.6$ & $\tau_{C}$ or $\zeta C$ & $t_{N}$ or $T_{N}$ & $\tau_{N}$ or $\varsigma_{N}$ & Health & Expenses \\
\hline no RP & 4.82 & 9.91 & 0.65 & 79.83 & 4.98 \\
RP & 6.01 & 10.02 & 1.32 & 78.57 & 1.88 \\
no RP & 5.88 & 9.86 & 1.22 & 79.11 & 5.09 \\
RP & NO & 9.49 & 1.52 & 78.12 & 6.39 \\
\hline
\end{tabular}

From the simulation we conclude that:

1. The reference pricing system tends to delay innovations. The only case where this not the case is when the level $C$ innovation disappears and the pioneer arrives sooner. 


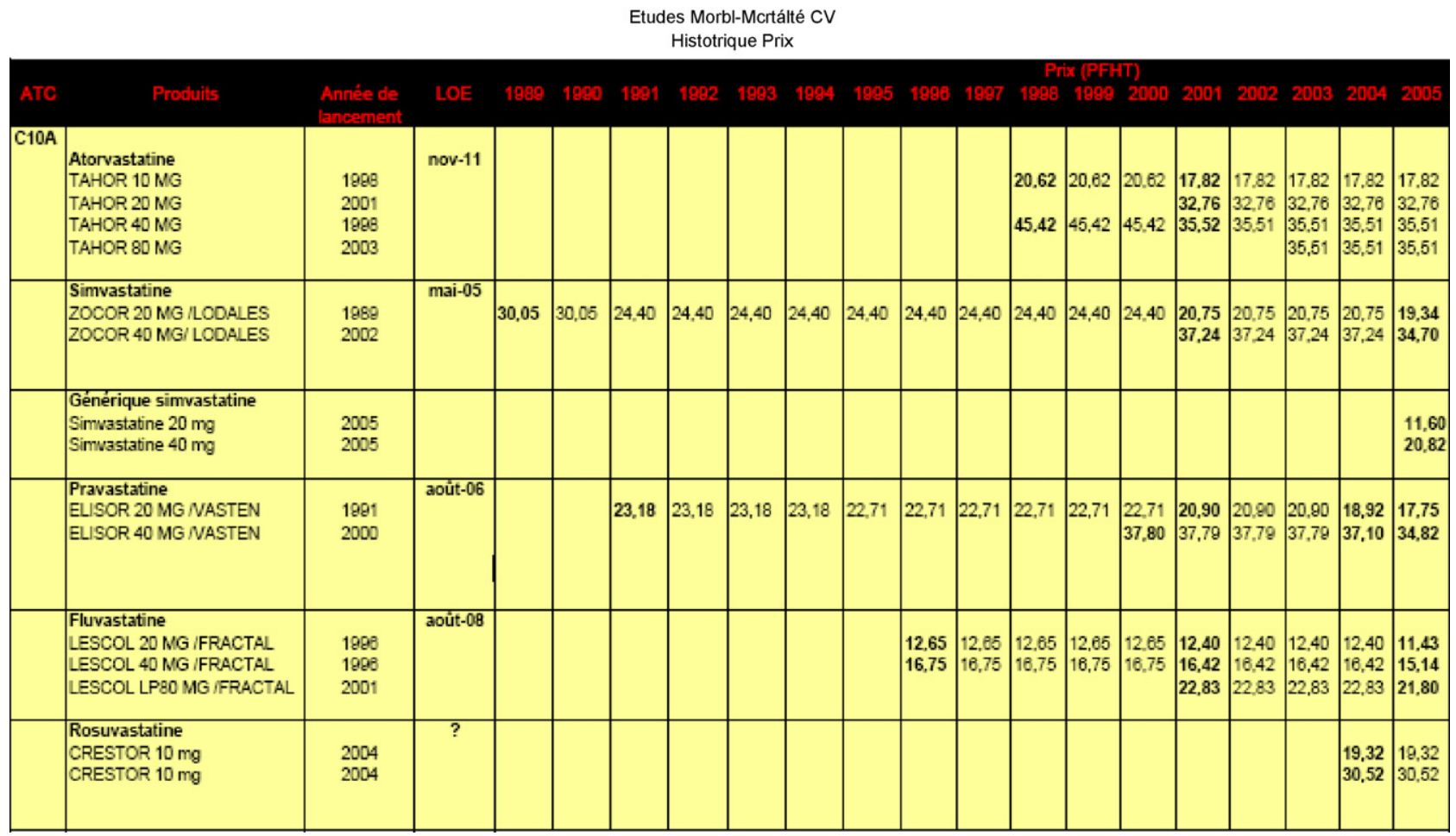

Fig. 3. Statins prices.

2. In all cases, the reference pricing system has a negative impact on health surplus.

3. The system reduces expenses when the trajectory of innovation is not affected due to lower prices and delayed innovations.

4. But it raises expenses when some innovation is discouraged.

The global welfare effect will depend on the weight put on expenses relative to health. In any case it is negative when the trajectory changes.

\section{Extensions}

We have investigated two specific extensions of the model that we report here. One concerns the objective of the pharmaceutical company, the other introduces some copayments (paid by patients). ${ }^{33}$ We provide a heuristic discussion dealing with these two issues. For the discussion that follows, we should have in mind the following remark. Suppose that the objective of the firm's negotiator is changed from $p_{j}^{i}$ to some function $p_{j}^{i} g\left(p_{j}^{i}\right)$. Then a simple revealed preference argument shows that the negotiated price is unchanged if $g($.$) is a constant, while it decreases if g($.$) is decreas-$ ing, and it increases if $g($.$) increases. ^{34}$

\subsection{Forward-looking producers}

In the negotiation game, we assumed that negotiators are shortsighted. We discuss here what happens if the firm's negotiator maximizes discounted profit.

\footnotetext{
33 A technical addendum dealing with the copayment issue is available upon request.

34 This conclusion is valid for several bargaining games we investigated. In the case of the Nash Bargaining problem used in our model, the objective function is multiplicative in nature so that any constant $g($.$) would be factored out.$
}

Consider the case of the introduction of a follower. In level $N$ there will be no innovation in the future. In level $C$, at the time of the negotiation, the date of the next pioneer can be infered by the firm and is predetermined. Roughly speaking, this date corresponds to the delay chosen at date zero by the laboratory investing in the pioneer. The discounted retail profit is then $p_{j}^{2}\left(1-e^{-r \Delta}\right) / 2 r$ where $\Delta$ is the anticipated delay before the next innovation. Because $\Delta$ is exogenous when the negotiation takes place, the non-myopic negotiator would negotiate the same price as a myopic negotiator.

Consider the case of the pioneer. In the pharmaceutical industry, there is a lapse of time between a new innovation being made public, and the negotiation of the price. ${ }^{35}$ Thus, we may start with the assumption that the R\&D investment in the follower occurs prior to price negotiation. Then in the case without reference pricing, the non-myopic negotiator would negotiate the same price as a myopic one. Indeed, at the negotiation stage, the delay of the follower is exogenous and the profit is proportional to the price. Thus, without reference pricing the same equilibrium emerges irrespective of whether the negotiator is myopic or not.

For the case with reference pricing we need to account for the effect of the pioneer's introductory price on the future reference price. Profit writes as

p. $\left(\frac{1-\delta^{\tau}}{1-\delta}+\frac{\delta^{\tau}}{1-\delta} \frac{1}{2}(1-\alpha)\left(1+\frac{\theta}{3 p}\right)\right)$.

Because the right term decreases with $p$, the non-myopic laboratory would negotiate a lower price than the myopic negotiator. The intuition is that the reference price is only partially affected by the introductory price of the pioneer. As the negotiator cares about the mean intertemporal price and not the current price, he is a softer bargainer.

35 This is because drugs have to go through several tests and approval stages before their introduction. 
Overall, allowing for a non-myopic negotiator adds a negative effect of reference pricing on the price of the pioneer, with more delays of both the pioneer and its follower. This would favour long sequences.

An additional effect arises if the price negotiation occurs prior to the R\&D investment in the follower, as negotiators internalize the effect of the introductory price on the date of introduction of the follower. Negotiated prices would be lower with and without reference pricing, but we were not able to sign the relative effects on profits and introductory delays.

\subsection{Copayment}

Assume that there is a small copayment $\beta$ for the patient. First, notice that nothing is changed for the negotiation of the introductory price of the pioneer as a small copayment would not affect demand.

In the duopoly case, adapting the proof of Lemma 1 , we find that for $\beta$ small (compared to $P+v+\theta / 6$ ), each firm sets its price at its introductory level without reference pricing, ${ }^{36}$ and at the reference price with reference pricing. Thus, the relationship between the negotiated prices and the market prices remains unchanged. However, the consumers' allocation when two drugs are available in the market changes. More precisely, with a copayment, there is a shift of demand toward the cheapest drug. This implies higher consumers' welfare and higher profit for the follower, at given prices.

Let us consider the negotiation of the price for a follower without reference pricing. Compared to a situation in which patients do not pay copayment, one can show that the welfare is relatively more sensitive to a price reduction. At the same time, the profit of the follower is relatively less affected by a price reduction because the increase in demand mitigates the impact of a price reduction. Thus the introductory prices of followers is lower in the case with no reference pricing. The same reasoning can be adapted to show that the welfare gain and the short run profit of the follower would both be higher with copayment than without.

Another consequence is that the introductory price and the profit would be lower for the pioneer in a long sequence: indeed the regulator would start with a higher welfare pre-negotiation, and the next follower is introduced faster.

To sum-up, with a copayment and no reference pricing, prices would be lower, while a long sequence would be more likely. By contrast, nothing would be changed in the case with reference pricing because demand is spread evenly between the two laboratories in the duopoly case.

\section{Conclusions}

The model emphasizes the interaction between the pricing policy constrained by various forms of regulation and the effort of innovation by pharmaceutical laboratories. We have identified antagonist effects of the reference pricing regulation: a decrease in price reduces the incentives to create pioneer drugs; inversely, the introduction of followers is delayed, which gives positive incentives to launch pioneers. Consequently, the net effect of the uniform pricing rule within a class is ambiguous.

\footnotetext{
${ }^{36}$ For $\left|p_{1}-p_{2}\right| \leq \theta / \beta$, the demand of each firm is positive and equal to $D_{i}=$ $\left(1-\beta / \theta\left(p_{i}-p_{j}\right)\right)^{2}$. Otherwise only one firm sells.
}

Simulations show that the dynamic impact on the health of the population is negative due to less innovation, while the impact on expenses is ambiguous. What appears is that by favouring pioneer innovations at the expense of cost reducing innovations, the regulation may generate a medium/long run increase in expenses, despite potential short run benefits.

While they need to be confirmed for different institutional setups, the mechanisms identified are clear and, in our view, quite robust in institutional details.

Our work also points to a more balanced view on the link between innovation and expenses. Innovation is a process that contributes both to cost inflation and to cost reduction. While the current heath policy debate has focused on the control of short run expenses, our analysis points to the fact that much more attention should be devoted to the role of innovation and its long run consequences.

Of course much remains done as many issues related to reference pricing remains.

In our model, we consider that reference pricing only embraces horizontally differentiated drugs. However, as we mention in the introduction, in some countries, reference pricing has been applied to vertical innovations as well. It could be an interesting extension to allow for vertical differences inside a therapeutic class. Indeed, DiMasi and Paquette (2004) mention the fact that, between 1960 and 1998, approximately one-third of me-toos received a priority rating from the FDA.

A related topic(s) where our approach should apply is the introduction of generics at the end of patent protection. Clearly the model can be extended to include a patent duration and to handle the problem of generic products. One key issue with generics, is that we do not have a satisfactory theory explaining the slow diffusion of generics observed in practice. It crucially depends on the institutional context and the rents let to actors such that physicians and pharmacists to operate the substitution between brand-name drugs and generics. Moreover, in our framework, it is worth noticing that branded drugs and generics would be vertically differentiated. Indeed, at the same price, patients tend to buy the branded drug.

On the innovation side, we have adopted a deterministic innovation model which fits somewhat the introductory efforts of the pharmaceutical firms. ${ }^{37}$ A complementary approach would be to account for uncertainty in research and in particular to analyze the retail market regulation from a risk sharing perspective where risk is shared between research institutions and society.

\section{Acknowledgements}

The authors thank Jacques Crémer, Richard Manning, Neal Masia for useful comments, as well as two anonymous referees and participants to ECHE (2006) and PEPC (2006). The authors benefited from the advice of Pfizer on the institutional and economic context, and research support, but note that the opinion expressed is the sole responsibility of the authors.

\footnotetext{
37 We have also ruled out pharmaceutical groups that develop several drugs and would internalize the effect of a new drug on the profitability of their portfolio. At the level of therapeutic equivalence class, this seems to be a reasonable assumption as groups tend to have only one molecule in a would-be class, but it ignores life cycle strategies where new patented drugs replace out of patent drugs.
} 


\section{Appendix A.}

Proof of Lemma 1. Let $p$ be the price of drug 1 , where $p_{j}^{1} \geq p \geq p_{j}^{2}$. The producer of drug 1 chooses to align the price of drug 1 to $p_{j}^{2}$ if $\max _{p_{j}^{2} \leq p \leq p_{j}^{1}} p . \operatorname{Prob}\left\{\tilde{x}_{j}^{1}-\tilde{x}_{j}^{2} \geq \frac{p-p_{j}^{2}}{\theta}\right\}=\frac{p_{j}^{2}}{2}$.

The demand is $D_{1}=0$ for $p \geq p_{j}^{2}+\theta$. Using $\operatorname{Prob}\left\{\tilde{x}_{j}^{1}-\tilde{x}_{j}^{2} \geq Z\right\}=$ $(1-Z)^{2} / 2$ for $0 \leq Z \leq 1$, the demand is

$D_{1}=\frac{1}{2}\left(1-\frac{p-p_{j}^{2}}{\theta}\right)^{2}$ for $p_{j}^{2} \leq p \leq p_{j}^{2}+\theta$.

We thus the condition:

$\max _{p_{j}^{2} \leq p \leq \inf \left\{p_{j}, p_{j}^{2}+\theta\right\}} p\left(1-\frac{p-p_{j}^{2}}{\theta}\right)^{2}=p_{j}^{2}$.

which holds if $\theta<2 p_{j}^{2}$.

Proof of Lemma 3. The derivative of $\chi(p)$ is

$\chi^{\prime}(p)=1-\frac{1}{2}\left(1+\frac{p}{p+(\theta / 3)}\left(\frac{r}{\gamma-r}\right)\right)\left(\frac{(1-\alpha)[p+(\theta / 3)]}{c 2 r}\right)^{r /(\gamma-r)}$.

$\chi(p)$ is concave and by Assumption 2, for $p$ in the relevant range

$$
\begin{aligned}
\chi^{\prime}(p) & >1-\frac{1}{2}\left(1+\frac{p}{p+(\theta / 3)}\left(\frac{r}{\gamma-r}\right)\right) \\
& >\frac{1}{2} \frac{p((\gamma-2 r) /(\gamma-r))+(\theta / 3)}{p+(\theta / 3)} .
\end{aligned}
$$

This is positive for $\gamma>2 r$ for any price.

Proof of Lemma 4. The function is

$\Xi(p)=p-\frac{1}{2}\left(\frac{(1-\alpha)[p+(\theta / 6)]}{c 2 r}\right)^{r /(\gamma-r)}\left(p(1+\alpha)-(1-\alpha) \frac{\theta}{6}\right)$.

The derivative of $\Xi(p)$ is

$$
\begin{aligned}
\Xi^{\prime}(p)= & 1-\frac{1}{2}\left(1+\alpha+\frac{p(1+\alpha)-(1-\alpha)(\theta / 6)}{p+(\theta / 6)}\left(\frac{r}{\gamma-r}\right)\right) \\
& \times\left(\frac{(1-\alpha)[p+(\theta / 6)]}{c 2 r}\right)^{r /(\gamma-r)} .
\end{aligned}
$$

By Assumption 2

$$
\begin{aligned}
\Xi^{\prime}(p) & >1-\frac{1}{2}\left(1+\alpha+\frac{p(1+\alpha)-(1-\alpha)(\theta / 6)}{p+(\theta / 6)}\left(\frac{r}{\gamma-r}\right)\right) \\
& >(1-\alpha) \frac{1}{2}\left(\frac{\gamma}{\gamma-r}\right) \frac{p(1-(2 r /((1-\alpha) \gamma)))+(\theta / 6)}{p+(\theta / 3)} .
\end{aligned}
$$

This is positive for $(1-\alpha) \gamma>2 r$ for any price.

Proof of Proposition 1 and Corollary 1. The first point follows from $P_{N}^{2}<p_{N}^{2}$. For the second point, $T_{N}>t_{N}$ iff

$$
\begin{aligned}
& \left(\frac{(1-\alpha)\left[p_{N}^{1}+(\theta / 6)\right]}{c 2 r}\right)^{r /(\gamma-r)}\left(p_{N}^{1}-\frac{1}{2}(1-\alpha)\left[p_{N}^{1}+\frac{\theta}{6}\right]\right) \\
& >\left(\frac{(1-\alpha)\left[p_{N}^{1}+(\theta / 3)\right]}{c 2 r}\right)^{r /(\gamma-r)} \frac{p_{N}^{1}}{2}
\end{aligned}
$$

which writes as

$2-(1-\alpha)\left[1+\frac{\theta}{6 p_{N}^{1}}\right]>\left(1+\frac{1}{\left(6 p_{N}^{1} / \theta\right)+1}\right)^{r /(\gamma-r)}$.

Moreover, as $p_{N}^{1}=(1-\alpha)[P+v]$, we have

$1+\alpha>\frac{\theta}{6[P+v]}+\left(1+\frac{1}{(((1-\alpha) 6[P+v]) / \theta)+1}\right)^{r /(\gamma-r)}$.

Which is true if $P$ is high enough.

The RHS decreases with $v$ and increases with $\theta$ and $r$, which implies the results stated in Corollary 1.

Proof of Proposition 2. The level-1 follower is not introduced if $\max _{\tau<t_{N}(k)} \frac{p_{C}^{2}(l)}{2 r}\left(e^{-r \tau}-e^{-r t_{N}(k)}\right)-c e^{-\gamma \tau}<0$.

Let us denote $\Delta=t_{N}(k)-\tau$ the length of existence of the drug in level 1 if introduce at $\tau$ and define $\Phi(\Delta)=\left(c e^{(\gamma-r) \Delta}\right) /\left(1-e^{-r \Delta}\right)$. Then the condition of no introduction can be rewritten writes as $\min _{\left[0, t_{N}(k)\right]} \Phi(\Delta) \geq \frac{p_{C}^{2}(l)}{2 r} e^{(\gamma-r) t_{N}(k)}$.

The slope of the function $\Phi$ is $\Phi^{\prime}(\Delta)=\Phi(\Delta)\left(\gamma-\left(r /\left(1-e^{-r \Delta}\right)\right)\right) . \Phi$ is quasi-convex with a minimum

$\underline{\Phi}=\frac{c \gamma((\gamma-r) / \gamma)^{-((\gamma-r) / r)}}{r}$ at $\underline{\Delta}=-\frac{\ln (1-(r / \gamma))}{r}>0$.

By Assumption 2, we have

$\underline{\Phi}>\frac{p_{C}^{2}(l)}{2 r} e^{(\gamma-r) \underline{\Delta}}$.

If $t_{N}(k) \leq \underline{\Delta}, \Phi(\Delta)>\underline{\Phi}>\left(p_{C}^{2}(l) / 2 r\right) e^{(\gamma-r) \underline{\Delta}}>\left(p_{C}^{2}(l) / 2 r\right) e^{(\gamma-r) t_{N}(k)}$ for all $\Delta \leq t_{N}(k)$. Thus there is no introduction. If $t_{N}(k)>\Delta$, there is no introduction if $\left(p_{C}^{2}(l) / 2 r\right) e^{(\gamma-r) t_{N}(k)} \leq \Phi$, or if $t_{N}(k) \leq \overline{\bar{t}}=1 /(\gamma-$ $r) \ln \left[2 r \Phi / p_{C}^{2}(l)\right]$. The threshold is then

$\bar{t}=\frac{\ln c}{\gamma-r}-\frac{1}{\gamma-r} \ln \left[\frac{p_{C}^{2}(l)}{2 \gamma}\left(\frac{\gamma-r}{\gamma}\right)^{(\gamma-r) / r}\right]$

which gives the first result. An equilibrium exists for all values of $\bar{t}$ if $\bar{t}_{N}(s) \leq \bar{t}_{N}(l)$. When $\chi$ is increasing the results follows from $p_{N}^{1}(s)>$ $p_{N}^{1}(l)$.

Proof of Corollary 2. For $C$, this is immediate. For the effect of $\theta$ we can write

$$
\begin{aligned}
& (\gamma-r)\left(\bar{t}-t_{N}(s)\right) \\
& \quad=\ln \left(\frac{[P+v]\left(2-(((1-\alpha)[(1-\alpha)[P+v]+(\theta / 3)]) / c 2 r)^{r /(\gamma-r)}\right)}{P+(\theta / 3)}\right)
\end{aligned}
$$

which decreases with $\theta$. Then

$$
\begin{aligned}
& (\gamma-r)\left(\bar{t}-t_{N}(l)\right) \\
& \quad=\ln \left(\frac{(((2-\alpha) / 2) P+v-\alpha(\theta / 6))\left(2-(((1-\alpha)[((2-\alpha) / 2) P+(v /(1+\lambda))+(\theta / 3)(1-(\alpha / 2))]) / c 2 r)^{r /(\gamma-r)}\right)}{P+(\theta / 3)}\right)
\end{aligned}
$$

is also decreasing with $\theta$. 
When $v$ increases, $\bar{t}$ is not affected and the price $p_{N}^{1}(k)$ increases, hence the result for $\chi($.$) increasing.$

When $\alpha$ increases $\bar{t}-t_{N}(s)$ increases, while the effect on $\bar{t}-t_{N}(l)$ is ambiguous.

Proof of proposition 4. Same as for Proposition 2 with the new prices. The function $\chi(p)$ becomes $\Xi(p)$. The delay for the follower in level $N$ is given as before while the introductory delay of the pioneer is now given by

$T_{N}(k)=\frac{1}{\gamma-r}\left(\ln (C)-\ln \frac{1}{r}\left[\Xi\left(P_{N}^{1}(k)\right)\right]\right)$.

We find that

$\bar{T}=\frac{\ln c}{\gamma-r}-\frac{1}{\gamma-r} \ln \left[\frac{P_{C}^{2}(l)}{2 \gamma}\left(\frac{\gamma-r}{\gamma}\right)^{(\gamma-r) / r}\right]>\bar{t}$,

where the inequality follows from $P_{C}^{2}(l)<p_{C}^{2}(l)$.

Proof of Corollary 4. For the sequence short we have

$\frac{\left(p_{N}^{1}(s)\right)(1+\alpha)}{p_{N}^{1}(s)}=1+\alpha>\left(\frac{p_{N}^{1}(s)}{p_{N}^{1}(s)}\right)^{r /(\gamma-r)}=1$.

This extend to the sequence long, as the price is smaller. Moreover

$\bar{T}-\bar{t}=\frac{1}{\gamma-r}\left(\ln \left[\frac{p_{1}^{2}(l)}{P_{1}^{2}(l)}\right]\right) ;$

$T_{N}(k)-t_{N}(k)=\frac{1}{\gamma-r}\left(\ln \left[\frac{\chi\left(p_{N}^{1}(k)\right)}{\Xi\left(P_{2}^{1}(k)\right)}\right]\right)$.

Thus $\bar{T}-\bar{t}>T_{N}(s)-t_{N}(s)$ iff $p_{1}^{2}(l) / P_{1}^{2}(l)>\chi\left(p_{N}^{1}(s)\right) / \Xi\left(p_{N}^{1}(s)\right)$. When $\theta=0$, we have $\tau_{N}(s)=\varsigma_{N}(s)$ and the condition writes

$\frac{P}{P(1-(\alpha / 2))}>\left[\frac{p\left(2-e^{-r \tau_{N}(s)}\right)}{p\left(2-e^{-r \tau_{N}(s)}(1+\alpha)\right)}\right]_{p=(1-\alpha)[P+v]}$

which reduces to

$1>\frac{3}{2} e^{-r \tau_{N}(s)}$,

and gives the condition.

Computations of welfare. In the absence of reference pricing, and when innovation follows a short sequence, we have

$$
\begin{aligned}
& h(s)=e^{-r t_{N}(s)} v+e^{-r\left(t_{N}(s)+\tau_{N}(s)\right)} \frac{\theta}{6} \\
& e(s)=e^{-r t_{N}(s)}\left(p_{N}^{1}(s)-P\right)+e^{-r\left(t_{N}(s)+\tau_{N}(s)\right)}\left(\frac{p_{N}^{2}(s)-p_{N}^{1}(s)}{2}\right) .
\end{aligned}
$$

For a long sequence, we have

$$
\begin{aligned}
h(l)= & e^{-r \tau_{C}(l)} \frac{\theta}{6}+e^{-r t_{N}(l)}\left(v-\frac{\theta}{6}\right)+e^{-r\left(t_{N}(l)+\tau_{N}(l)\right)} \frac{\theta}{6} \\
e(l)= & e^{-r \tau_{C}(l)}\left(\frac{p_{C}^{2}(l)-P}{2}\right)+e^{-r t_{N}(l)}\left(p_{N}^{1}(l)-\frac{P+p_{C}^{2}(l)}{2}\right) \\
& +e^{-r\left(t_{N}(l)+\tau_{N}(l)\right)}\left(\frac{p_{N}^{2}(l)-p_{N}^{1}(l)}{2}\right) .
\end{aligned}
$$

In the presence of reference pricing, health expenditures are modified due to the price alignment. Thus we obtain:

$$
\begin{aligned}
E(s)= & e^{-r T_{N}(s)}\left(P_{N}^{1}(s)-P\right)+e^{-r\left(T_{N}(s)+\varsigma_{N}(s)\right)}\left(P_{N}^{2}(s)-P_{N}^{1}(s)\right) ; \\
E(l)= & e^{-r \zeta_{C}(l)}\left(P_{C}^{2}(l)-P\right)+e^{-r T_{N}(l)}\left(P_{N}^{1}(l)-P_{C}^{2}(l)\right) \\
& +e^{-r\left(T_{N}(l)+\varsigma_{N}(l)\right)}\left(P_{N}^{2}(l)-P_{N}^{1}(l)\right) .
\end{aligned}
$$

\section{References}

Aghion, P., Tirole, J., 1994. Opening the black box of innovation. European Economic Review.

Anderson, S., de Palma, A., Thisse, J., 1992. Discrete Choice Theory of Product Differentiation. MIT Press.

Barro, R., Sala-i-Martin, X., 1995. Economic Growth. MIT Press.

Berndt, E.R., Cockburn, I.M., Grepin, K., 2006. The Impact of Incremental Innovation in Biopharmaceuticals: Drug Utilization in Original and Supplemental Indications. mimeo MIT.

Brekke, K., Konigbauer, I., Straume, O., 2007. Reference pricing of pharmaceuticals. Journal of Health Economics 26, 613-642.

Brekke, K., Kuhn, M., 2006. Direct to consumer adverstising in pharmaceutical markets. Journal of Health Economics 25 (1), 102-130.

Danzon, P.M., 2001. Reference pricing: theory and evidence. In: Lopez-Casasnovas, G., Jonsson, B. (Eds.), Reference Pricing and Pharmaceutical Policy. Springer, New York.

Danzon, P., Ketchman, J., 2004. Reference pricing of pharmaceuticals for medicare: evidence from Germany, the Netherlands and New Zeland, Frontiers in Health Policy Research 7:2, bepress.

Danzon, P., Liu, H., 1996. Reference pricing and physician drug budgets: the German experience in controlling pharmaceutical expenditures. Working paper, University of Pennsylvania.

Danzon, P., Pauly, M., 2002. Health insurance and the growth in pharmaceutical expenditures. Journal of Law and Economics XLV, 587-613.

DiMasi, J.A., 2000. New drug innovation and pharmaceutical industry structure: trends in the output of pharmaceutical firms. Drug Information Journal 34, 1169-1194.

Dimasi, J.A., Hansen, R.W., Grabowski, H.G., 2003. The price of innovation: new estimates of drug development costs. Journal of Health Economics 22, 151-185.

Dimasi, J.A., Hansen, R.W., Grabowski, H.G., Lasagna, L., 1991. Cost of innovation in the pharmaceutical industry. Journal of Health Economics 10 (2), 107-142.

DiMasi, J.A., Paquette, C., 2004. The economics of follow-on drug research and development. Pharmacoeconomics 22 (Supl 2), 1-14.

Dranove, D., Metzler, D., 1994. Do important drugs reach the market sooner. Rand Journal of Economics 25, 402-422.

Gal-Or, E., 1997. Exclusionary equilibria in health care markets. Journal of Economic and Management Strategy, 5-43.

Gal-Or, E., 1999. Mergers and exclusionary practices in health care markets. Journal of Economic and Management Strategy, 315-350.

Hurwitz, M., Caves, R., 1988. Persuasion or information? Promotion and the shares of brand-name and generic pharmaceuticals. Journal of Law and Economics 31 299-320.

Laffont, J.-J., Tirole, J., 1993. A Theory of Incentives in Procurement and Regulation. MIT Press.

Lopez-Casanovas, G., Puig-Junoy, J., 2000. Review of the literature on reference pricing. Health Policy 54, 87-123.

O'Donoghue, T., Scotchmer, S., Thisse, J., 1998. Patent breadth, patent life, and the pace technological progress. Journal of Economic and Management Strategy 7 (1), 1-32. 